\title{
Vehicle-Mounted Self-Organizing Network Routing Algorithm Based on Deep Reinforcement Learning
}

\author{
Shitong Ye $\mathbb{D}^{1},{ }^{1}$ Lijuan $\mathrm{Xu}^{1},{ }^{1}$ and Xiaomin $\mathrm{Li} \mathbb{D}^{2}$ \\ ${ }^{1}$ Department of Data Science, Guangzhou Huashang College, Guangzhou 511300, China \\ ${ }^{2}$ Department of Mechanical and Electrical Engineering, Zhongkai University of Agriculture and Engineering, \\ Guangzhou 511300, China
}

Correspondence should be addressed to Shitong Ye; yst888_0@126.com

Received 6 March 2021; Revised 22 May 2021; Accepted 9 June 2021; Published 1 July 2021

Academic Editor: Wei Wang

Copyright (c) 2021 Shitong Ye et al. This is an open access article distributed under the Creative Commons Attribution License, which permits unrestricted use, distribution, and reproduction in any medium, provided the original work is properly cited.

Through the research on the vehicle-mounted self-organizing network, in view of the current routing technical problems of the vehicle-mounted self-organizing network under the condition of no roadside auxiliary communication unit cooperation, this paper proposes a vehicle network routing algorithm based on deep reinforcement learning. For the problems of massive vehicle nodes and multiple performance evaluation indexes in vehicular ad hoc network, this paper proposes a time prediction model of vehicle communication to reduce the probability of communication interruption and proposes the routing technology of vehicle network by studying the deep reinforcement learning method. This technology can quickly select routing nodes and plan the optimal route according to the required performance evaluation indicators.

\section{Introduction}

With people's continuous concern about the intelligent and safety of vehicles, the current artificial intelligence technology related to vehicle-driverless technology has emerged. Driverless technology can replace the human's own operation of the vehicle [1-3], so that the human can liberate their hands when using the vehicle for walking. Driverless technology makes the car react faster when encountering danger in the process of driving, to ensure the safety of passengers $[4,5]$. Although the main research on driverless technology of major technology companies is to solve the problem of automatic control of vehicles, it does not mean that driverless technology only has the application field of intelligent vehicle control [6-9]. Vehicles with driverless technology can also be equipped with sensors and communication systems to cruise according to the set route in the military theater. While building the vehicular ad hoc network, due to the use of driverless technology, it can also prevent the enemy from causing casualties when attacking our vehicular system [10-12]. Using driverless technology to build a vehicle self-organizing network, it can also be deployed to extreme environments such as desert and disaster areas to perform tasks such as environmental data, animal and plant data collection, emergency rescue, and disaster relief information contact, etc. And through the vehicle network, the relevant data will be transmitted to the terminal, and the processing and decision-making can be carried out at the terminal, which can reduce the manpower input and avoid the safety problems of personnel in the execution of tasks. When unmanned vehicles are used to build communication networks in war zones or extreme environments with limited communication, technical problems are not only the automatic cruise technology of unmanned vehicles but also the deployment of vehicle networks and the realization of communication routes [13-15].

Vehicular ad hoc network, due to the fast-moving speed of vehicle nodes, the topology of the link will change dramatically in the process of network deployment, the network connection state of some vehicle nodes in the network will continue to change, the communication link will be interrupted, and it is unable to build an effective communication route. Therefore, routing technology is a major technical problem of vehicular ad hoc network. Moreover, vehicles carrying fixed fuel in theater or extreme environment will face 
the problem of insufficient fuel when performing tasks and may not be able to continuously supply energy for on-board communication nodes. When the energy is insufficient, node death will cause topology change, communication link interruption, and data transmission interruption. Therefore, when performing data transmission tasks, it is necessary to consider the energy problem of the transmitting node. When data is transmitted through an effective routing node, according to the transmission rate of the node, the energy of the routing node can be maintained for a period of time until the data is successfully transmitted [16-18]. To solve the abovementioned vehicle self-organizing network routing technology problem, this paper proposes an adaptive routing technology in the vehicle self-organizing network based on the research of deep neural network technology, which can consider energy consumption and data transmission efficiency indicators, and automatically construct node data transmission route to complete the successful transmission of task data.

\section{Related Work}

In the research field of the routing technology of the vehicle ad hoc network, many researchers have done a lot of research work on the transmission efficiency and performance optimization of the multihop routing of the vehicle network. Zhang et al. proposed a link duration model based on duration, which can evaluate link reliability and use it as a key parameter for designing a new routing protocol. The new routing protocol can dynamically adjust the routing path through interaction with the surrounding environment [19]. Li et al. proposed an optimization model for the lowcarbon vehicle routing problem under multigraph timevarying networks. The researchers started from the study of the multipath attributes of the real road network and designed a time division method that conforms to the timevarying network carbon emission calculations. The impact of driving speed changes and vehicle load on emissions, a low-carbon vehicle path optimization model under a multichannel time-varying network, is established [20]. Researchers such as Ahmed et al. proposed a highly secure QoS-aware routing algorithm that uses an optimal trust management scheme. Multihop clusters are implemented through an improved whale optimization algorithm, and the trust value is used to complete intercluster routing. This method can quickly discover routes and reduce the packet loss rate [21]. Silva proposes a new routing protocol called adaptive, which considers routing performance indicators such as transmission rate, average delay, and average number of hops. The protocol is based on a predictable connection concept and uses the history of meeting nodes to determine the best way to route and discard data packets on the network, and this method can effectively improve the transmission efficiency of routing data [22]. David and Vanathi proposed a vehicle-mounted self-organizing network clustering model that reduces data packet loss and uses clustering algorithms to cope with frequent topology changes and high mobility of vehicle networks, manage vehicles in an effective way, and provide intervehicle uninterrupted communication
[23]. Meng et al. designed a control strategy for official vehicles in the traffic road network to improve the $K$-means algorithm to make the nodes of the official vehicle network adapt to the route, increase the weight of the backpressure strategy according to traffic pressure conditions, and improve the parameters through optimization the adaptability of official vehicles in the traffic road network [24]. Beirigo et al. proposed a dual-mode vehicle routing in hybrid autonomous and nonautonomous regional networks and introduced a new mathematical programming model in the routing to achieve coordinated routing planning for autonomous and conventional vehicles [25].

\section{Time Prediction Model for Vehicular Ad Hoc Networks}

For the vehicle-mounted ad hoc network without the cooperation of the roadside auxiliary communication unit, the biggest problem is that the vehicle communication link may be interrupted at any time due to the change of the vehicle speed, which greatly affects the communication quality of the vehicle ad hoc network. Therefore, in the research of vehicle network routing technology, we must first consider the problem of link interruption in vehicle self-organizing network [26, 27]. According to the sensing range of onboard sensors, the distance of end-to-end vehicle nodes, vehicle speed, vehicle speed variation range, task packet size, and network transmission rate, this paper proposes a time prediction model, which is used for the current vehicle. The node predicts a batch of candidate next hop nodes [28, 29]. The candidate nodes selected by the time prediction model meet certain conditions, that is, when the current vehicle node selects these candidate nodes as the relay point of the next hop, the probability of link interruption is small [30, 31]. Therefore, the time prediction model needs to comprehensively consider the transmission time of task packets, sensing range, vehicle spacing, vehicle speed, and possible speed mutation. As shown in Figure 1, the model will select candidate nodes that meet the time prediction function. It can be seen from the schematic diagram in Figure 1 that car $\mathrm{A}$ is the current node, and it is assumed that the range that car A's on-board sensor can sense is within the range of the black circle. Among them, car B, car C, and car D are the candidate nodes selected by car A. Although other nodes are also in the sensing range of car A, they do not meet the requirements of time prediction function, so they cannot be used as candidate nodes.

Assuming that the vehicle car A needs to select the next hop node to forward the data packet, the sensing radius of car $\mathrm{A}$ is $R$, and the initial interval between it and a certain vehicle (assuming car B) is $L_{0}\left(L_{0}<R\right)$. The communication time for car A to transmit complete data is $t$. The time prediction model is used to judge whether car B can be used as a candidate node for the next hop: let all vehicles drive at a constant speed, acceleration, or deceleration, and the maximum acceleration is $\alpha_{\max }$. The conditions for car B to be a candidate node for the next hop are as follows: if car A runs at a constant speed with the minimum speed $v_{0}$, car B runs at a constant speed with the initial speed $v_{0}$ and the maximum 


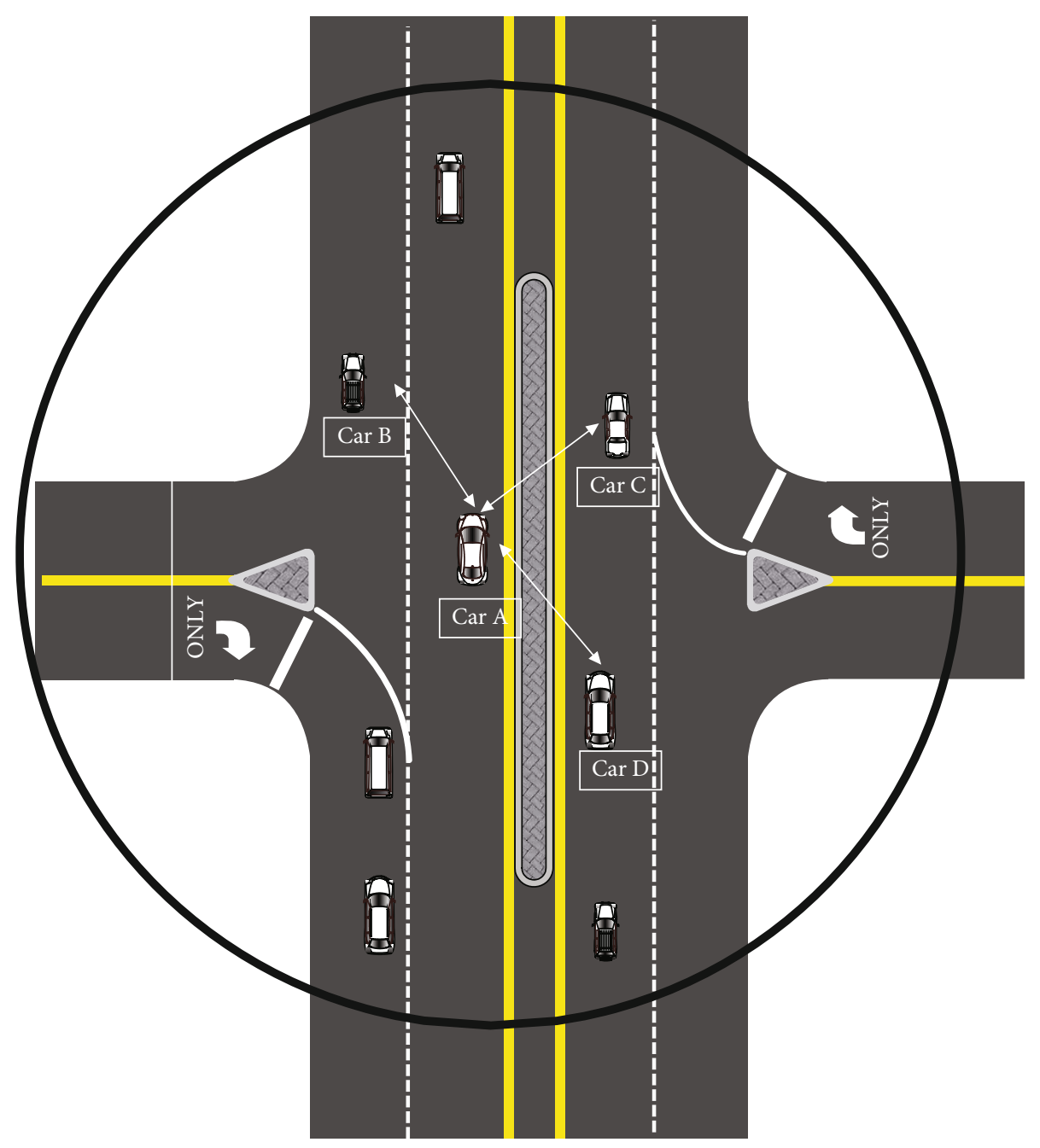

Figure 1: Schematic diagram of current node selection candidate node.

acceleration $\alpha_{\max }$, and after $t$ time, car B is still in the sensing radius $R$ of car $\mathrm{A}$, then car $\mathrm{B}$ can be a candidate node for car A's next hop.

First, the relationship between driving distance and time of car A and car B is calculated:

$$
\left\{\begin{array}{l}
S_{A}(t)=\int_{0}^{t} v_{0} d x, \\
S_{B}(t)=\int_{0}^{t} v_{0}+a_{\max } d x .
\end{array}\right.
$$

After $t$ seconds, the distance relationship between car A and car B satisfies the following relationship, then car B can be the next hop candidate node of car A:

$$
S_{B}(t)-S_{A}(t)+L_{0} \leq R \text {. }
$$

\section{Utility Functions of Candidate Vehicle Routing Nodes}

For the current vehicle node $x 1$, in order to successfully transmit the data packet to $y 1$ through the relay node, as shown in Figure 2, it is assumed that $x 1$ calculates the candidate nodes of its next hop through the time prediction model, and these nodes from a candidate node set as $N_{A}, N_{A}=\left\{n_{1}\right.$, $\left.n_{2}, \cdots, n_{a}\right\}$. After selecting the next hop node $n_{i}\left(n_{i} \in N_{A}\right)$ of vehicle node $x 1$ from the candidate node set $N_{A}, n_{a}$ continues to calculate its next hop candidate node through the time prediction model, assuming that the candidate node set is $M_{B}, M_{B}=\left\{m_{1}, m_{2}, \cdots, m_{b}\right\}$. Select the next hop node $m_{j}\left(m_{j}\right.$ $\in M_{B}$ ) from the set $M_{B}$ until the data is finally transmitted to $y 1$.

Selecting the best relay node from the candidate node set of each hop can constitute the multihop optimal routing of vehicle network. In this paper, deep reinforcement learning is used to select nodes from each candidate node set. In order to train in the deep reinforcement learning algorithm, it is necessary to determine the reward (or penalty) of the current system under the node selection behavior. In this section, we use the comprehensive utility function to evaluate the reward (or punishment) of node selection behavior.

The comprehensive utility function mainly considers the energy loss and transmission rate of data transmission between nodes. Suppose that in the vehicular network, the vehicular sensor nodes all adopt wireless communication 


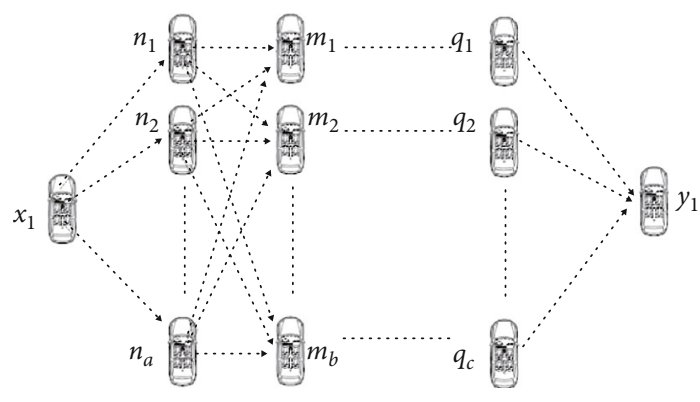

FIGURE 2: Schematic diagram of vehicle network packet transmission route.

mode, the distance between the current node and the next hop node is $L$, and the loss of link energy is represented by $\operatorname{Loss}(L)$. Considering the attenuation of transmission link, the $\operatorname{Loss}(L)$ is as follows:

$\operatorname{Loss}(L)=40 \log L-10\left(\log G_{l}+\log G_{r}\right)-20\left(\log h_{l}+\log h_{r}\right)$,

where $G_{l}$ is the antenna gain of the vehicle sensor, $G_{r}$ is the antenna gain of the receiver, $h_{l}$ is the antenna height of the transmitter, and $h_{l}$ is the antenna height of the receiver.

Assuming that the transmitting power of the current node is $h_{l}$ and the unit noise power is $P_{c}$, the direct data transmission rate between the current node and the next hop node is

$$
v=\log _{2}\left(1+\frac{P_{T}}{P_{c} n_{0}}\right),
$$

where $n_{0}$ is interference noise.

The comprehensive utility function of the current node and the next hop node is expressed as:

$$
\mathrm{RE}=\frac{v}{P_{T}+\operatorname{Loss}(L)}
$$

The higher the transmission rate and the lower the link loss between the current node and the next hop node, the larger the comprehensive utility function.

\section{Vehicle Routing Based on Deep Reinforcement Learning}

In vehicle network, the system state only considers the channel state of nodes. In Figure 2, the vehicular sensor node $x 1$ transmits data to $y 1$ through the relay node, and the node switches to the next state by selecting the relay node. We model the relay node selection scheduling problem as a Markov decision process.

In the Markov decision function, the next state $S_{t+1}$ of the system is only related to the current state $S_{t}$

$$
P_{s s^{\prime a}}=P\left(S_{t+1}=\dot{s} \mid S_{t}=s, A_{t}=a\right) .
$$

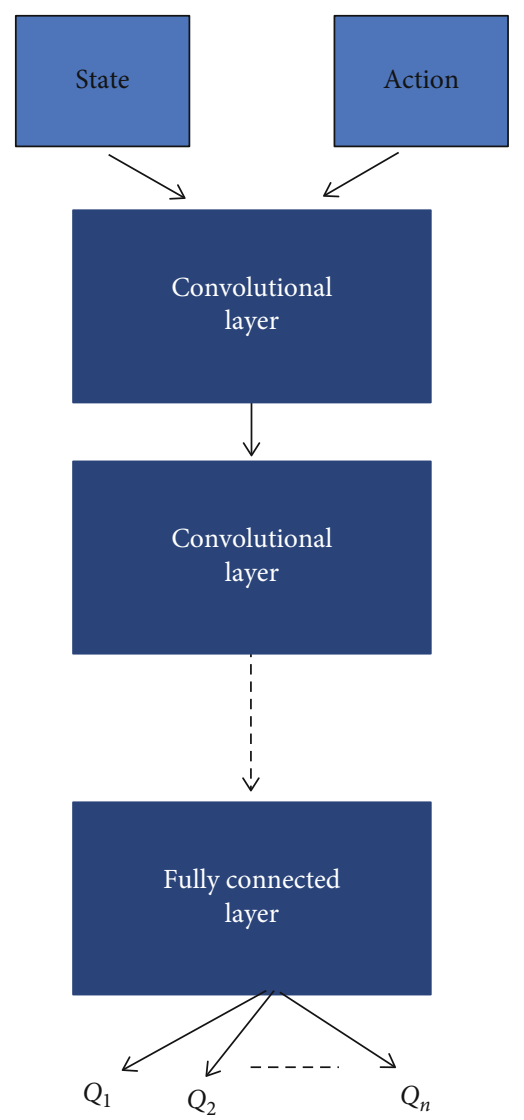

Figure 3: Obtaining $Q$ value through deep network.

Let $A_{x 1}=\left\{a_{t, 1}, a_{t, 2}, \cdots, a_{t, n}\right\}$ be the next hop candidate node set of the starting node $x 1$ in time slot $t$, that is, $A_{x 1}$ is also the action set in time slot. $a_{t, i}\left(a_{t, i} \in A_{x 1}\right)$ means that the $i$-th node is selected as the relay node from the candidate node set of $x 1$ in time slot $t$.

The current system performs node selection in state $S_{t}$. In order to successfully transmit data from the original node $x 1$ to $y 1$, assuming that $k$ relay nodes are needed, the profit of the future $k$ steps is obtained through the state value function:

$$
V_{\pi}(s)=E_{\pi}\left[\sum_{t=0}^{k} \gamma^{t} \mathrm{RE}_{t+1} \mid S_{t}=s\right] .
$$

Among them, $\gamma^{t}$ is the discount factor at step $\gamma^{t}$, and $\mathrm{RE}_{t+1}$ represents the comprehensive utility function value of the current node and the selected next hop node.

In order to evaluate the state and behavior of the system, the $Q_{\pi}(s, a)$ value is used to represent the action value function:

$$
Q_{\pi}(s, a)=E_{\pi}\left[\sum_{t=0}^{k} \gamma^{t} Q_{\pi}\left(s_{t+1}, a ́\right) \mid S_{t}=s, A_{t}=a\right]
$$

Combined with the state value function, the following action value function is used to evaluate the system's profitability: 
Select multi-hop routing for vehicle network based on deep reinforcement learning

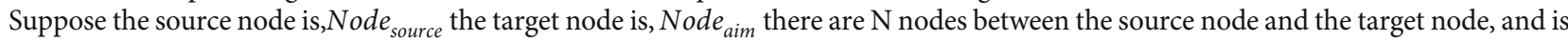
the node set.

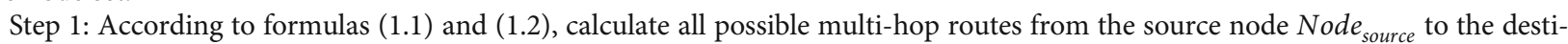
nation node Node $_{\text {aim }}$ to form a candidate multi-hop route set $R_{N}$.

Step 2:Calculate the comprehensive utility value of all candidate multi-hop routes in $R_{N}$ according to formula (1.5).

Step 3:The comprehensive utility value is used as a reward, and according to formulas (1.6)-(1.12), deep reinforcement learning is used to adaptively select the best multi-hop route.

Algorithm 1: Algorithm implementation steps.

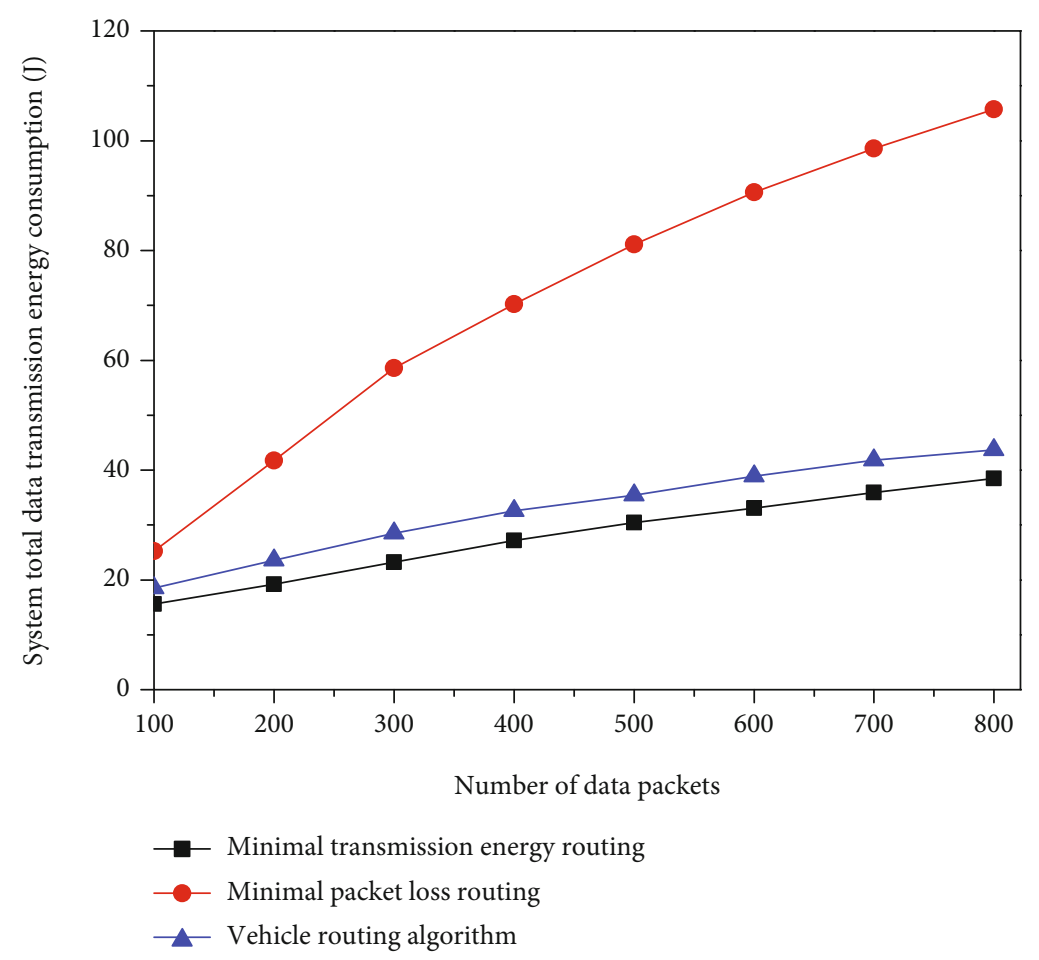

FIgURE 4: Total energy consumption of system transmission.

$$
Q_{\pi}(s, a)=R_{s}^{a}+\gamma \sum_{s \in S} P_{s s^{\prime a} V_{\pi}(\dot{s})}
$$

Among them, $R_{s}^{a}$ represents the sum of rewards accumulated in all states after performing a set of actions.

In order to obtain better node selection behavior data in the network, iterative update is required, and iterative formulas are used to achieve optimized learning of action value functions:

$$
\left\{\begin{array}{l}
Q_{\pi}\left(s_{t}, a_{t}\right)=Q_{\pi}\left(s_{t}, a_{t}\right)+\lambda B_{\pi} \\
B_{\pi}=R_{s}^{a}+\gamma Q_{\pi}\left(s_{t+1}, a_{t+1}\right)-Q_{\pi}\left(s_{t}, a_{t}\right)
\end{array}\right.
$$

Among them, $\lambda$ represents the learning rate, and $\gamma$ represents the impact of future returns on current behavior.

Because the traditional Q-learning reinforcement learning method is based on the past state, statistics, and iterative $Q$ value. Therefore, the state and action space applicable to $Q$ -learning is very small, and if a state never appears, $Q$ -learning cannot handle it. Therefore, here we use a deep reinforcement learning algorithm to replace the $Q$ table with a neural network to obtain the $Q$ value corresponding to the state and action. According to the state and node selection behavior, the $Q$ value of each node selection action is output through the convolutional layer and the fully connected layer, as shown in Figure 3.

The optimization objective of deep reinforcement learning is to minimize the loss

$$
\text { Loss }=E\left[\left(R_{s}^{a}+\gamma Q_{\pi}\left(s_{t+1}, a_{t+1}\right)-Q_{\pi}\left(s_{t}, a_{t}\right)\right)^{2}\right] \text {. }
$$

The gradient descent method is used to update the weight

$\frac{\partial \operatorname{Loss}(w)}{\partial w}=E\left[\left(R_{s}^{a}+\gamma Q_{\pi}\left(s_{t+1}, a_{t+1}\right)-Q_{\pi}\left(s_{t}, a_{t}\right)\right) \frac{\partial Q_{\pi}\left(s_{t}, a_{t}, w\right)}{\partial w}\right]$. 


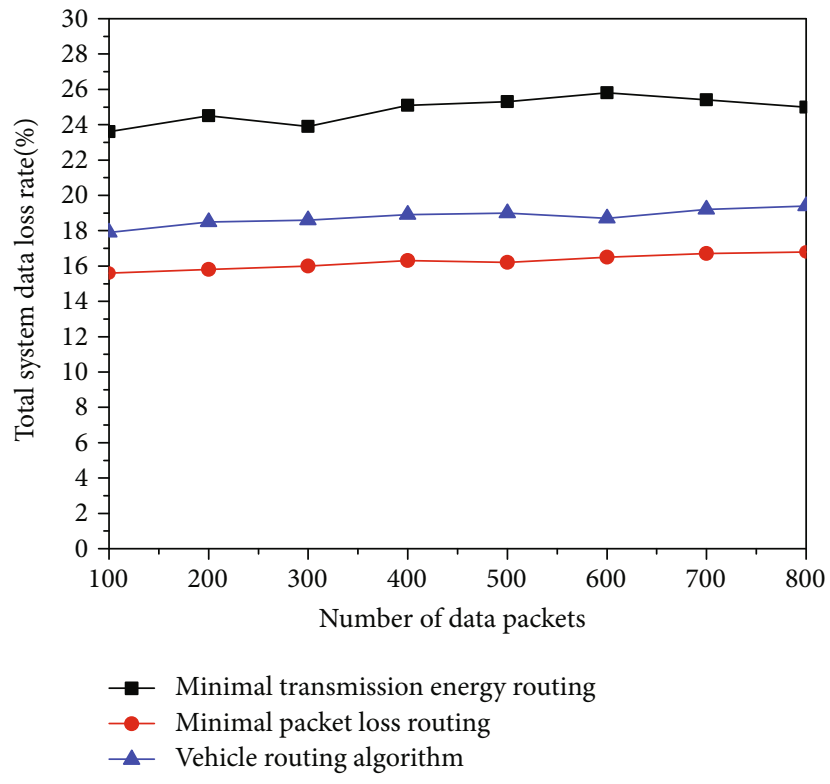

FIGURE 5: System transmission packet loss rate.
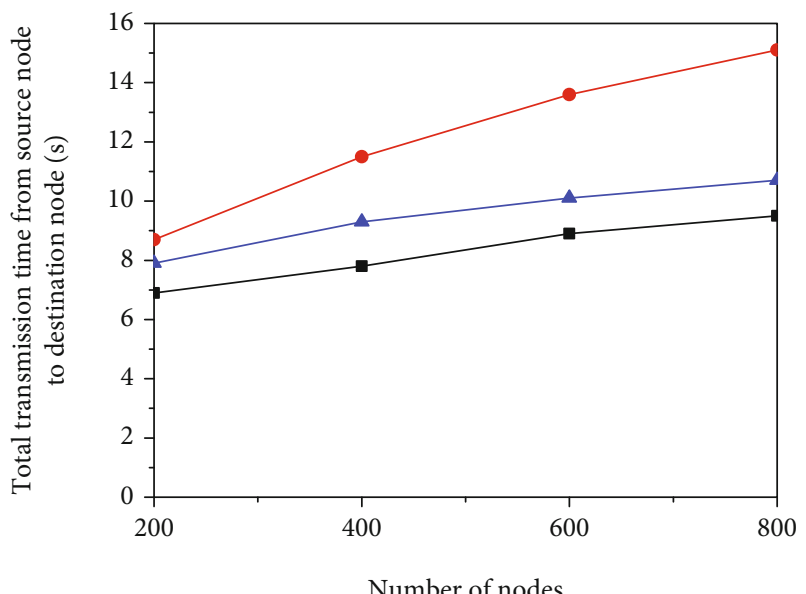

- - Minimal transmission energy routing
- Minimal packet loss routing
- - Vehicle routing algorithm

Figure 6: The total time for the source node to transmit data to the destination node.

The deep reinforcement learning method is used to update the $Q$ value iteratively, so that the vehicle network can adaptively construct the optimal multihop routing from the source node to the destination node according to the comprehensive utility value.

The algorithm implementation process is shown in Algorithm 1:

\section{Simulation Results}

In the simulation experiment, there are 200 vehicular nodes in the simulated vehicular ad hoc network scenario. The nodes use wireless communication and data relay mode. Assuming that the computing power of the nodes is suffi- cient, the computing time is ignored, the noise power is set as $P_{c}=1 \times 10^{-2} \mathrm{~W}$, the discount factor is $\gamma=0.6$, and the training error of the deep network is lower than Loss $=1 \times$ $10^{-4}$. In the process of simulation, the simulated road is a straight passage, the width of the road is 16 meters, the width of the vehicle node is 2 meters, the set minimum speed of the vehicle is $20 \mathrm{~km} / \mathrm{h}$, and the maximum speed is $110 \mathrm{~km} / \mathrm{h}$.

In order to verify the performance of this algorithm, we set up two contrast algorithms in the experiment, one is to select the routing node according to the method of minimum transmission energy consumption, and the other is to select the routing node according to the method of minimum transmission packet loss rate. The two algorithms are tested under the same conditions as the algorithm in this paper.

In order to verify the performance of the proposed algorithm in terms of transmission energy consumption and packet loss rate, we conducted 100 simulation experiments and obtained the average results. In the simulation experiment of vehicular ad hoc network, we calculate the total transmission energy consumption and packet loss rate of the algorithm when the network system transmits 100 800 packets from random source node to random destination node.

According to the experimental statistical chart of the total energy consumption of the system transmission in Figure 4 and the experimental statistical chart of the packet loss rate of the system transmission in Figure 5, the more packets are transmitted, the greater the total energy consumption of the system, and the packet loss rate of the system has no obvious change trend with the increase of the packets. Among them, the minimum energy routing method can better save the system transmission energy consumption, but the system packet loss rate is higher, because the routing node selected by this method only considers the transmission link distance between the current node and the next hop node, so although it can better save the transmission energy consumption of the vehicle network, it will cause higher data packet loss. 


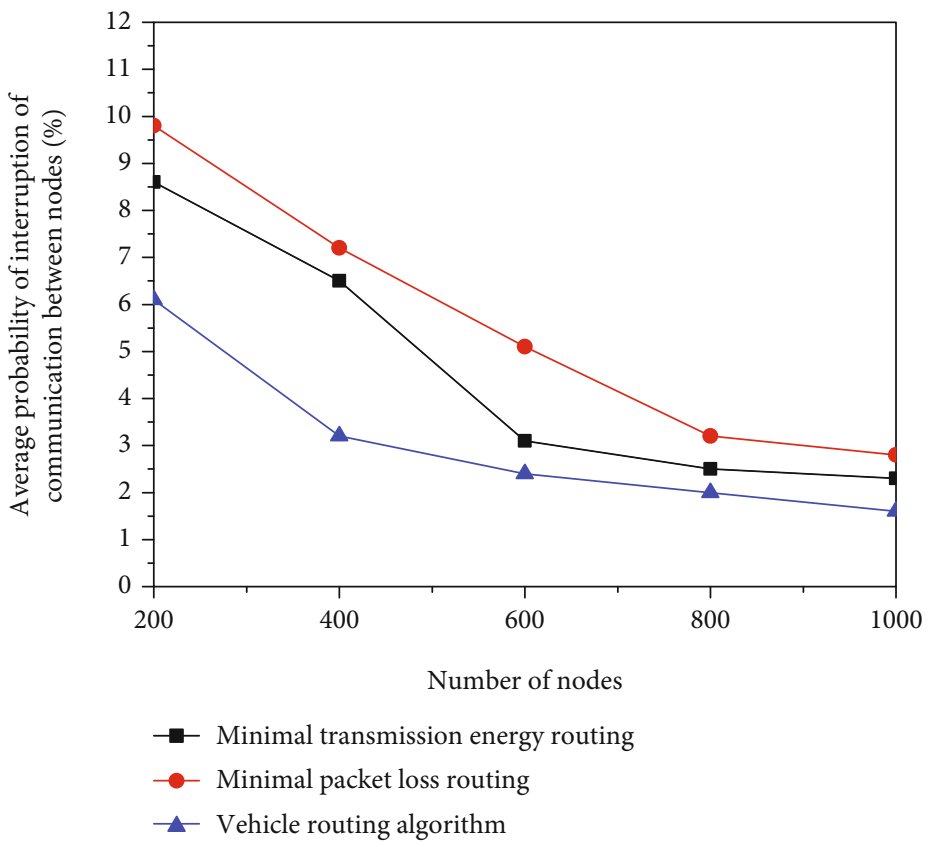

Figure 7: Average probability of communication interruption between nodes.

It can be seen from Figure 5 that the minimum packet loss routing can reduce the total packet loss rate of the system, but from the result of Figure 4, the total energy consumption of the minimum packet loss routing is more. However, the vehicle routing method based on deep reinforcement learning proposed in this paper selects the candidate next hop node according to the time prediction model, although there may be packet loss when the next hop node cannot be selected. However, from the experimental results in Figure 5, this method can maintain a low packet loss rate on the whole, and by using the comprehensive utility as the reward and punishment factor of deep reinforcement learning, the vehicle routing will further consider the transmission energy consumption when selecting nodes. From the results of Figure 5, this method can keep low transmission loss. Therefore, in general, the vehicle routing algorithm based on deep reinforcement learning proposed in this paper, although it cannot achieve the lowest transmission energy consumption and the lowest packet loss rate, is the best in the overall performance of the system transmission energy consumption and packet loss rate.

In order to verify the efficiency of the vehicle network routing algorithm proposed in this paper in data transmission, in the experiment, by increasing the number of experimental nodes, the time required for the vehicle network to transmit data from the random source node to the random destination node is counted. It can be seen from the experimental statistical results in Figure 6 that with the increase of the number of nodes, the time required to transmit data from the source node to the destination node increases gradually. Among them, the minimum energy routing method needs the least data transmission time. This is because the method adopts the shortest path data transmission method, which can quickly transmit the data to the destination node without considering the packet loss. The method in this paper uses the time prediction model, so it takes into account the risk of link interruption, and the transmission time is shorter than the minimum packet loss routing algorithm, which has obvious advantages.

In order to verify the performance of this algorithm in reducing the probability of communication interruption between nodes, in the experiment, we increase the total number of nodes tested and count the average probability of communication interruption between nodes in the vehicle network, as shown in Figure 7. From the figure, we can see that with the increase of vehicle nodes, the average outage probability of communication between nodes will continue to decrease, because the increase of vehicle nodes means that more nodes can act as relay nodes, reducing the possibility of link outage. It can be seen from the comparison in Figure 7 that the algorithm in this paper has a better effect on reducing the average probability of communication interruption between nodes. Because the method in this paper adopts the time prediction model, it can select the better next hop node to complete the data transmission task.

\section{Conclusion}

In order to reduce the probability of link interruption and improve the energy consumption and transmission efficiency of vehicular ad hoc network, a routing algorithm based on deep reinforcement learning is proposed in this paper. In this algorithm, a time prediction model of vehicular ad hoc network is proposed, which can effectively reduce the probability of communication interruption between vehicle nodes. The algorithm also uses the deep reinforcement learning method to select multihop routing, which can reduce the transmission loss of vehicle network routing and provide transmission efficiency. 


\section{Data Availability}

The data used to support the results of this study needs to be obtained with the consent of the corresponding author.

\section{Consent}

Informed consent was obtained from all individual participants included in the study references.

\section{Conflicts of Interest}

We declare that there is no conflict of interest.

\section{Acknowledgments}

This article is supported by Characteristic Innovation Project (Natural Science) in Colleges and Universities in Guangdong Province (No.: 2019KTSCX237) and Innovation Team Project of Humanities and Social Sciences in Colleges and Universities of Guangdong Province (No.: 2020WCXTD008).

\section{References}

[1] S. Liu, "Novel dynamic source routing protocol (DSR) based on genetic algorithm-bacterial foraging optimization (GABFO)," International Journal of Communication Systems, vol. 31, no. 18, article e3824, 2018.

[2] D. G. Zhang, G. Li, K. Zheng, X. Ming, and Z. H. Pan, "An energy-balanced routing method based on forward-aware factor for wireless sensor networks," IEEE Transactions on Industrial Informatics, vol. 10, no. 1, pp. 766-773, 2014.

[3] D. G. Zhang, S. Liu, T. Zhang, and Z. Liang, "Novel unequal clustering routing protocol considering energy balancing based on network partition \& distance for mobile education," Journal of Network and Computer Applications, vol. 88, no. 15, pp. 1-9, 2017.

[4] D. Zhang, X. Wang, X. Song, and D. Zhao, "A novel approach to mapped correlation of ID for RFID anti-collision," IEEE Transactions on Services Computing, vol. 7, no. 4, pp. 741748, 2014.

[5] J. Yang, M. Ding, G. Mao, Z. Lin, D. G. Zhang, and T. H. Luan, "Optimal base station antenna downtilt in downlink cellular networks," IEEE Transactions on Wireless Communications, vol. 18, no. 3, pp. 1779-1791, 2019.

[6] T. G. Dias, "Driverless cars - another piece of the puzzle: comments on "driverless cars will make passenger rail obsolete," by Yair Wiseman [Opinion]," IEEE Technology and Society Magazine, vol. 38, no. 2, pp. 36-38, 2019.

[7] E. Psyllou and J. Pawlak, "Congestion, safety, economic, and environmental challenges of vehicle automation in transport systems: comment on "driverless cars will make passenger rail obsolete," by Yair Wiseman [Opinion]," IEEE Technology and Society Magazine, vol. 38, no. 2, pp. 28-35, 2019.

[8] A. Zarkeshev and C. Csiszár, "Patients' willingness to ride on a driverless ambulance: a case study in Hungary," Transportation Research Procedia, vol. 44, pp. 8-14, 2020.

[9] L. Dong, D. Sun, G. Han, X. Li, Q. Hu, and L. Shu, "Velocityfree localization of autonomous driverless vehicles in underground intelligent mines," IEEE Transactions on Vehicular Technology, vol. 69, no. 9, pp. 9292-9303, 2020.
[10] A. Sharif, J. Guo, J. Ouyang et al., "Compact base station antenna based on image theory for UWB/5G RTLS embraced smart parking of driverless cars," IEEE Access, vol. 7, pp. 180898-180909, 2019.

[11] S. Liu, K. Hu, W. Ni, Z. Xu, F. Wang, and Z. Wan, “A cognitive relay network throughput optimization algorithm based on deep reinforcement learning," Wireless Communications and Mobile Computing, vol. 2019, Article ID 2731485, 8 pages, 2019.

[12] M. F. Baumann, C. Brandle, and S. Zimmer-Merkle, "Dis/orientations on driverless driving and autonomous vehicles?" Applied Mobilities, vol. 4, no. 2, pp. 251-255, 2019.

[13] R. Mumford, "Driverless cars technology receives 20 million boost in UK," Microwave Journal, vol. 59, no. 3, pp. 49-50, 2016.

[14] Z. Wang, Y. Guo, C. Fang, M. Li, Y. Sun, and Y. Zhang, "Optimal control strategy design with minimum energy consumption for connected vehicle systems," in 2019 IEEE Global Communications Conference (GLOBECOM), Waikoloa, HI, USA, 2020.

[15] P. Si, Y. He, H. Yao, R. Yang, and Y. Zhang, "DaVe: offloading delay-tolerant data traffic to connected vehicle networks," IEEE Transactions on Vehicular Technology, vol. 65, no. 6, pp. 3941-3953, 2016.

[16] A. Garcia-Santiago, J. Castaneda-Camacho, J. F. GuerreroCastellanos, and G. Mino-Aguilar, "Evaluation of AODV and DSDV routing protocols for a FANET: further results towards robotic vehicle networks," in 2018 IEEE 9th Latin American symposium on Circuits \& Systems (LASCAS), Puerto Vallarta, Mexico, 2018.

[17] T.-Y. Huang, C.-J. Chang, C.-W. Lin, S. Roy, and T.-Y. Ho, "Delay-bounded intravehicle network routing algorithm for minimization of wiring weight and wireless transmit power," IEEE Transactions on Computer-Aided Design of Integrated Circuits and Systems, vol. 36, no. 4, pp. 551-561, 2017.

[18] X. Chen, Z. Wei, H. Tian, and Z. Feng, "Space-time spectrum sharing for unmanned aerial vehicle networks," in 2018 IEEE/CIC International Conference on Communications in China (ICCC Workshops), Beijing, China, 2018.

[19] T. Zhang, T. Zhang, and X. Liu, "Novel self-adaptive routing service algorithm for application in VANET," Applied Intelligence, vol. 49, no. 5, pp. 1866-1879, 2019.

[20] L. Shunyong, D. Bin, and G. Xianlong, "Optimization model and algorithm of low carbon vehicle routing problem under multi-graph time-varying network," Computer Integrated Manufacturing System, vol. 25, no. 2, pp. 454-468, 2019.

[21] S. Ahmed, N. V. K. Ramesh, and B. N. K. Reddy, "A highly secured QoS aware routing algorithm for software defined vehicle ad-hoc networks using optimal trust management scheme," Wireless Personal Communications, vol. 113, no. 4, pp. 1807-1821, 2020.

[22] R. F. Silva, "Adaptive: an adaptive routing protocol for vehicle delay-tolerant networks," IEEE Latin America Transactions, vol. 18, no. 2, pp. 223-231, 2020.

[23] S. David and P. T. Vanathi, "Middle-order vehicle-based clustering model for reducing packet loss in vehicular ad-hoc networks," Journal of Circuits, Systems and Computers, vol. 29, no. 11 , pp. $7-15,2020$.

[24] X. Meng, J. Lv, and S. Ma, “Applying improved K-means algorithm into official service vehicle networking environment and research," Soft Computing, vol. 24, no. 11, pp. 8355-8363, 2020. 
[25] B. Beirigo, F. Schulte, and R. Negenborn, "Dual-mode vehicle routing in mixed autonomous and non-autonomous zone networks," in 2018 IEEE International Conference on Intelligent Transportation Systems (ITSC), Maui, HI, USA, 2018.

[26] S. Radley, C. J. Sybi, and K. Premkumar, "Multi information amount movement aware- routing in FANET: flying ad-hoc networks," Mobile Networks and Applications, vol. 25, no. 2, pp. 596-608, 2020.

[27] R. A. Nazib and S. Moh, "Routing protocols for unmanned aerial vehicle-aided vehicular ad hoc networks: a survey," IEEE Access, vol. 8, pp. 77535-77560, 2020.

[28] Z. Wan, Z. Pan, W. Ni et al., "A vehicle mobile internet of things coverage enhancement algorithm based on communication duration probability analysis," IEEE Access, vol. 7, pp. 98356-98365, 2019.

[29] A. Rawat, M. Khodari, M. Asplund, and A. Gurtov, "Decentralized firmware attestation for in-vehicle networks," $A C M$ Transactions on Cyber-Physical Systems, vol. 5, no. 1, pp. 123, 2020.

[30] G. A. Klunder, H. Taale, L. Kester, and S. Hoogendoorn, "Improvement of network performance by in-vehicle routing using floating car data," Journal of Advanced Transportation, vol. 2017, Article ID 8483750, 16 pages, 2017.

[31] M. W. Levin, M. Duell, and S. T. Waller, "Effect of road grade on networkwide vehicle energy consumption and ecorouting," Transportation Research Record: Journal of the Transportation Research Board, vol. 2427, no. 1, pp. 26-33, 2018. 